\title{
¿Qué hay tras la permanencia universitaria? Los cambios más significativos en el acompañamiento académico'
}

\section{Resumen}

Rodolfo Martinic Lenta ${ }^{2}$

https://orcid.org/0000-0001-7179-319X

El artículo presenta los resultados de una evaluación de impacto cualitativa de los servicios de apoyo académico que brinda la Universidad de Santiago de Chile a través del Programa de Acceso Inclusivo, Equidad y Permanencia (PAIEP). Este programa surge en el 2012 como una política de acción afirmativa que articula y fortalece las iniciativas orientadas al acceso, permanencia y titulación de la Universidad, teniendo dentro de sus funciones brindar apoyo académico a todos los estudiantes de primer año, con un foco especial en los que ingresan mediante vías de acceso inclusivo. El apoyo académico fue evaluado a través de una técnica de evaluación cualitativa denominada el cambio más significativo, que se basa en la recolección de historias sobre los cambios que han experimentado las personas cuando participan de una intervención social. Se llevaron a cabo cuatro talleres evaluativos con estudiantes que tuvieron una alta participación en los servicios de acompañamiento de PAIEP. Los resultados del estudio consisten en los cinco cambios principales que relataron los estudiantes: a) la generación de hábitos de estudio; b) la mejor comprensión de los contenidos de las asignaturas; c) la generación de confianza en sí mismo; d) el mejoramiento del desempeño académico y, por último, e) la contribución a la integración social y universitaria. Finalmente, en la discusión se aborda la contribución de la evaluación al estudio de los servicios de apoyo académico en la educación superior, así como las ventajas y limitaciones de la metodología utilizada.

\section{Palabras clave}

Educación superior - Primer año universitario - Soporte académico - El cambio más significativo.

1- Se agradece a Roxana Aranda por sus valiosos comentarios, a Alexia Vásquez por su apoyo en el levantamiento de información y a Cristóbal Fuentes por su colaboración en el análisis. Asimismo, se agradece Ministerio de Educación del Gobierno de Chile por el financiamiento en el marco de su programa MECESUP.

2- Universidad de Santiago de Chile, Santiago, Chile. Contacto: rodolfo.martinic.lenta@gmail.com. 


\section{What is behind higher-education permanence?}

\section{Abstract}

The article presents the results of a qualitative impact evaluation of the academic support services offered by the Universidad de Santiago, Chile, through the Programa de Acceso Inclusivo, Equidad y Permanencia (Program for Inclusive Access, Equality, and Permanence, PAIEP in its acronym in Spanish). This program arises in 2012 as an affirmative action policy that articulates and strengthens the initiatives aimed at access to, permanence in, and graduation at the university; its purpose is to provide academic support to all junior students, with a special focus on those whose admission was through inclusive access. The academic support was evaluated utilizing a qualitative assessment technique known as "the most significant change", which is based on the collection of stories about the changes people have experienced when participating in a social intervention. Four evaluation workshops were held with students who had a high involvement in the PAIEP follow-up services. The main findings of the study consist in five changes reported by the students: a) the acquisition of study habits, b) the understanding of the academic subjects, c) the generation of self-confidence, d) the improvement of academic performance, and e) the contribution to social integration. Finally, the discussion addresses the contribution of evaluation to the study of academic support services in higher education, as well as the advantages and limitations of the methodology employed.

\section{Keywords}

Higher education - Tirst-year student - Academic support - The most significant change.

\section{Introducción}

Una de las transformaciones que a nivel mundial ha experimentado la educación superior en las últimas décadas se relaciona con el tránsito desde instituciones de élite a sistemas masificados, una verdadera democratización en el acceso al saber que ha significado el ingreso creciente de estudiantes no tradicionales y la configuración de un cuerpo estudiantil más heterogéneo que en el pasado (en términos socioeconómicos, étnicos, de género, de edad, etc.) (WARREN, 2002; 0CDE, 2009). En la actualidad, los estudiantes que ingresan a la educación superior son más diversos en cuanto a su origen social, sus experiencias y antecedentes educativos, lo que implica que no siempre cuentan con la misma preparación para enfrentar la alta demanda académica, lo cual se traduce en altas tasas de deserción particularmente durante el primer año académico (SILVA, 2011).

Dentro de los estudios sobre permanencia en la universidad, es posible identificar dos corrientes de investigaciones principales que contrastan entre sí. Por un lado, a partir de la década de los 70', especialmente en Estados Unidos, un importante grupo de investigaciones buscaron generar modelos para explicar la deserción estudiantil y las causas que se encuentran a la base del abandono durante el crítico primer año (TINTO, 
1993; PASCARELLA; TERENZINI, 1980; BEAN, 1980). Esta línea de trabajo representa el paradigma dominante y se ha caracterizado por un posicionamiento epistemológico positivista y por el uso de metodologías cuantitativas con el objetivo de identificar las características individuales para predecir la permanencia y el éxito académico de los estudiantes (BENSIMON, 2007).

Por otro lado, a partir de los 80' en la sociología francesa surgen un conjunto de trabajos que desde diferentes enfoques teórico-metodológicos (fenomenología, etnometodología, etc.) abordan la situación de los estudiantes en el contexto de la universidad de masas. Bajo nociones como las de afiliación (COULON, 1997), condición (FELOUZIS, 2001) o experiencia estudiantil (DUBET, 1994), las investigaciones muestran desde la perspectiva de los propios estudiantes los cambios que experimentan en el inicio de la socialización universitaria. Particularmente, en el trabajo de Coulon $(1997,2017)$ la permanencia en la universidad es entendida como un proceso de afiliación por medio del cual el recién ingresado se encamina a alcanzar el oficio de estudiante. La entrada a la universidad, así, se asocia a la adquisición progresiva de un nuevo status social que se alcanza por medio del desarrollo de la autonomía, la incorporación de los códigos del nuevo entorno social y el establecimiento de un vínculo con el saber disciplinar, lo que genera una afiliación tanto institucional como intelectual en el estudiante.

Tomando distancia de la primera corriente -aunque reconociendo sus aportesy situándose más cerca de la segunda, el presente estudio consiste en una evaluación cualitativa de las medidas de apoyo académico para la permanencia que brinda la Universidad de Santiago de Chile a los estudiantes de primer año, tarea que se ve alentada por dos razones principales. En primer lugar, un importante corpus de evaluaciones de programas que buscan promover la permanencia y el éxito académico de los estudiantes en la universidad afirma -entre otras cosas- la escasa relevancia otorgada a la indagación en la percepción de los participantes sobre la influencia del programa (JACOBI,1991; CRISP; CRUZ, 2009; GERSHENFELD, 2014). En segundo lugar, pese a que parte importante de las instituciones de educación superior de Chile cuentan con programas enfocados en la permanencia de los estudiantes de primer año, estos han sido poco estudiados y es reconocida la falta de evidencia en cuanto a su funcionamiento (SCHEELE, 2015).

Por las características de la metodología utilizada, el cambio más significativo (DAVIES; DART, 2005), la evaluación realizada se centró en recomponer las experiencias de los estudiantes en la intervención a partir de los cambios que señalan haber vivido y que atribuyen a su participación en el programa. En ese sentido, constituye más un esfuerzo de generar una cartografía de los cambios posibles que pueden experimentarse en un programa de apoyo académico universitario, que un intento de garantizar su efecto en la permanencia por medio de la cuantificación de su impacto.

El artículo se estructura de la siguiente manera. En primer lugar, se describe el contexto de la educación superior en Chile con el fin de situar el Programa de Acceso, Inclusivo, Equidad y Permanencia (PAIEP) de la Universidad de Santiago. En segundo lugar, se expone la metodología del estudio, haciendo especial énfasis en la técnica de evaluación de impacto utilizada, el cambio más significativo. En tercer lugar, se exhiben los resultados del estudio, que corresponden a los cinco cambios principales identificados por los estudiantes. Finalmente, se discuten los resultados obtenidos en vistas del aporte 
al estudio de la permanencia desde la perspectiva desarrollada por Coulon $(1997,2017)$, así como del alcance de la metodología utilizada.

\section{Antecedentes}

\section{Los problemas en el acceso a la Educación Superior en Chile}

La educación superior en Chile ha crecido exponencialmente en los últimos veinte años, ha experimentado una masificación de la matrícula que ha significado el tránsito desde un 14\% de cobertura en 1990 a un 60\% en el 2016 (CARREÑO; MICIN; URZUA, 2016). Sin embargo, uno de los principales desafíos que enfrentan las universidades en la actualidad se relaciona con la diversidad y equidad en el acceso. En efecto, la heterogeneidad socioeconómica del estudiantado de las universidades chilenas -especialmente de las más selectivas- se ve comprometido debido a que los estudiantes provenientes de los sectores de menores recursos obtienen peores resultados en la Prueba de Selección Universitaria (PSU) (OCDE, 2009).

La PSU constituye el instrumento de selección del sistema único de admisión al cual se encuentran adscritas la mayoría de las universidades chilenas y es aplicada en todo el territorio nacional. La evidencia generada en torno a esta, sin embargo, señala que refleja la inequidad del sistema educativo del país, presentando una brecha socioeconómica más alta que la observada a nivel internacional (KOLJATIC; SILVA, 2010). De esta forma, el nivel socioeconómico junto a la dependencia del establecimiento de educación básica y media, son aspectos que inciden en la continuación de estudios en la educación superior de los estudiantes chilenos. A lo anterior, cabe añadir que los planes de estudio y los perfiles de egreso de cada formación diferenciada (Humanista-Científico o Técnico-Profesional) "[...] definen un camino más propenso hacia la educación superior universitaria y otro más propenso hacia el mercado laboral junto a una inserción precaria a la educación" (TREVIÑO et al., 2016, p. 11).

En este contexto, es posible entender que parte de las recomendaciones de la OCDE (2009) para el sistema de educación superior chileno apuntaran hacia la consideración de prácticas de discriminación positiva que incluyesen otros criterios adicionales a la PSU en la admisión, así como también en la asignación de cupos. Precisamente, la Universidad de Santiago de Chile tiene una importante tradición de medidas que avanzan en esa línea, por medio del desarrollo de vías de acceso inclusivo que permitan el ingreso de estudiantes con alto mérito académico provenientes de establecimientos de bajo nivel socioeconómico a la institución, así como en la entrega de apoyo para garantizar su permanencia y éxito académico.

\section{El Programa de Acceso Inclusivo, Equidad y Permanencia (PAIEP) de la Universidad de Santiago de Chile}

Por más de un cuarto de siglo, la Universidad de Santiago de Chile se ha caracterizado por su compromiso con la promoción de la igualdad de oportunidades de acceso a la educación superior, generando medidas dirigidas a posibilitar el ingreso de 
estudiantes con alto rendimiento escolar provenientes de escuelas de sectores de bajo nivel socioeconómico. Así, entre los hitos más importantes cabe considerar, en primer lugar, que entre los años 1992 y 2004 implementó una bonificación en el puntaje de postulación a los estudiantes con promedio de calificaciones pertenecientes al 15\% superior de la generación escolar. Luego, en el año 2007 comenzó a llevar a cabo el Propedéutico ${ }^{3}$, un programa de acceso cuya finalidad consistió en preparar académicamente en el contexto universitario a estudiantes de escuelas de bajo nivel socioeconómico durante su último semestre escolar, garantizándoles el acceso al programa de Bachillerato en Ciencias y Humanidades, de manera independiente del puntaje obtenido en la PSU. Esta iniciativa fue el referente principal del Programa de Acompañamiento y Acceso Efectivo (PACE) que el Ministerio de Educación comenzó a implementar en el 2015.

En el año 2012 se crea el PAIEP, una política de acción afırmativa que articula y fortalece las iniciativas generadas en la institución orientadas hacia el acceso, permanencia y titulación de estudiantes de alto rendimiento en contexto con el propósito de fortalecer sus recursos personales y nivelando sus competencias académicas (RAHMER; MIRANDA; GIL, 2013). Este Programa es el encargado de brindar apoyo académico a todos los estudiantes de primer año de la Universidad de Santiago, pero especialmente a aquellos que ingresaron mediante una de las seis vías de acceso inclusivo con las que cuenta la institución.

Concretamente, el apoyo académico es entregado por el área de Servicios de Acompañamiento para el Aprendizaje y la Permanencia (SAAP) del PAIEP, el cual consta principalmente de tres instancias:

- Tutorías: consisten en un acompañamiento orientado principalmente a los estudiantes que ingresan mediante vías de acceso inclusivo, en el cual tutores de tercer año de universidad en adelante trabajan durante la semana con una duración mínima de 45 minutos en función de las necesidades académicas que van presentando.

- Talleres: están enfocados en todos los estudiantes de primer año y consisten en clases abiertas realizadas por tutores con el objetivo de repasar contenidos y solucionar dudas en asignaturas críticas específicas.

- Asesorías: al igual que los talleres, se dirigen a todos los estudiantes de primer año y se basan en la atención de dudas en una asignatura particular y en un horario establecido por parte de un tutor.

Aunque todos los servicios se caracterizan por ser voluntarios, una diferencia importante de los talleres y las asesorías en relación a las tutorías es que estos se encuentran abiertos a todos los estudiantes de primer año y, por tanto, no requieren de una asistencia constante. Las tutorías, en cambio, son un acompañamiento de carácter personalizado que se focaliza en los estudiantes que más lo requieren y en una modalidad de trabajo sistemática.

Durante el año 2016 ingresaron a la Universidad de Santiago un total 3.861 estudiantes, de los cuales 2.156 participaron en los servicios de acompañamiento de PAIEP, lo que equivale a una tasa de atención del 66\% de la cohorte de dicho año. De esa

3- Una experiencia brasilera similar a este programa tanto en su diagnóstico como en sus objetivos es el Programa de Formação Interdisciplinar Superior (ProFis) de la Universidad Estatal de Campinas. 
cifra, los que recibieron tutorías corresponden al 20\% (433 estudiantes), mientras que el 80\% (1.723 estudiantes) fue atendido mediante talleres y asesorías.

En relación a la permanencia, los resultados del PAIEP son en gran medida alentadores. En efecto, de los 300 estudiantes que tuvieron una frecuencia de asistencia igual o superior a 20 sesiones de tutorías, talleres y/o asesorías, el 81,0\% inscribió asignaturas al segundo año de universidad, mientras que en el caso del resto de los estudiantes de la cohorte $(n=3.561)$ ese porcentaje equivalió a $78,5 \%$. Si se considera que el solo hecho de que las proporciones hubieran sido equivalentes hubiese sido positivo, que sea superior para quienes participaron constantemente del PAIEP es un resultado todavía mejor. Aquello, por supuesto, invita a explorar cuáles fueron los cambios más significativos que vivieron los estudiantes en el proceso de intervención.

\section{Metodología}

\section{El cambio más significativo}

La metodología del estudio fue de carácter cualitativa y utilizó la técnica de evaluación conocida como el cambio más significativo (CMS) (DAVIES; DART, 2005). El CMS es una técnica de evaluación participativa, basada en la recolección de historias sobre los cambios que han vivido las personas, grupos o comunidades desde que comenzaron a participar de una intervención social. A diferencia de los métodos convencionales en donde los criterios de evaluación son preestablecidos por los ejecutores de la intervención, el CMS busca conocer los cambios que experimentaron los participantes en instancias de dialogo y deliberación sobre cuáles fueron los más significativos.

Las historias sobre los cambios se recogen en base a una simple pregunta dirigida a los participantes de la intervención: "Desde que han participado en este programa, ¿cuál es el cambio más importante que han vivido?” La técnica, en ese sentido, utiliza inicialmente una pregunta general y abierta con el objetivo de que los participantes puedan mencionar cambios que no estén necesariamente planificados o contemplados inicialmente en la intervención. Luego de que se recogen las historias de cambio, se deben realizar preguntas más precisas y vinculadas a ámbitos específicos en donde la intervención busca generar deliberadamente ciertos cambios (por ejemplo, el rendimiento académico). En ambos momentos, es preciso que los investigadores tomen un papel activo y sondeen acerca del cómo, cuándo y dónde los participantes experimentaron los cambios que relatan.

La técnica, por otro lado, también considera en los cambios negativos que eventualmente pueden experimentar los participantes de un programa. Así, aunque sus creadores señalan que por lo general las historias de cambio que surgen suelen ser positivas, también pueden relatarse experiencias negativas, que en algunas evaluaciones realizadas con el CMS se les ha denominado áreas a mejorar, para así entenderlas como posibilidades de mejora a partir de las cuales el programa pueda aprender y crear valor (DAVIES; DART, 2005). Lo importante, de todas formas, es que la pregunta por los cambios negativos permite al programa conocer posibles consecuencias no buscadas de su intervención y que no han sido identificadas. 


\section{Participantes}

Los participantes del estudio correspondieron a estudiantes que durante el año 2016 asistieron a los servicios de apoyo académico brindados por el PAIEP, es decir: tutorías, asesorías y talleres. Siguiendo la lógica de un muestreo por caso extremo (SCRIBANO, 2008), en el cual se buscan informantes que presentan una característica de forma exacerbada, se invitó a participar de la evaluación a los estudiantes que asistieron a los servicios con una frecuencia superior a la mediana. El objetivo de aquello fue recoger la percepción de los estudiantes que más utilizaron los servicios $\mathrm{y}$, por tanto, que pudiesen hablar con mayor propiedad de estos.

La invitación a participar fue extendida inicialmente a treinta estudiantes, en donde se les indicó que se trataría de una conversación grupal en torno a sus experiencias en el PAIEP, se realizaría en el horario de almuerzo en las dependencias de la Universidad y además se les entregaría una colación. Así, finalmente se realizaron cuatro talleres evaluativos, en las cuales se aplicó la metodología del CMS y que contaron con la participación de un total de veinte estudiantes. De estos, ocho correspondieron a mujeres y doce a hombres, mientras que doce ingresaron mediante alguna vía de acceso inclusivo de la institución y ocho mediante ingreso regular. Previo al inicio de la dinámica del CMS, se les solicitó a los estudiantes la firma de un consentimiento informado en donde, junto con garantizar el anonimato de los estudiantes y la confidencialidad de la información, se autorizó a grabar el diálogo.

\section{Análisis de la información}

Para analizar la información que se generó en los talleres evaluativos en donde se aplicó la técnica del CMS con los estudiantes que participaron de PAIEP, se utilizaron los procedimientos de codificación de la Teoría Fundamentada (STRAUSS; CORBIN, 2002). La codificación, en ese sentido, corresponde al proceso analítico de fragmentar la información con el fin de integrarla gradualmente en conceptos de mayor nivel de abstracción y capacidad comprensiva. Las historias de cambio que atribuyeron los estudiantes a PAIEP y que relataron en los talleres evaluativos, fueron analizadas a través de los procedimientos de codificación abierta y axial.

\section{Resultados}

Los cambios más significativos que los estudiantes atribuyeron a PAIEP en base a la participación que tuvieron en los servicios de apoyo académico durante su primer año universitario, fueron cinco: a) la generación de hábitos de estudio; b) la mejor comprensión de los contenidos de las asignaturas; c) la recomposición de la confianza en sí mismo; d) el mejoramiento del desempeño académico y, por último, e) la contribución a la integración social y universitaria. A continuación, se expone la descripción y el análisis de cada uno de ellos. 
En relación a los cambios negativos, los estudiantes señalaron tres aspectos: por un lado, la estrechez de los espacios de estudio y falta de recursos para todos los estudiantes que acuden al programa y, por otro, la incompatibilidad de horarios con los servicios programados como los talleres. No obstante, si bien aquello corresponde a aspectos que pueden ser mejorados, no constituyen un cambio negativo que los estudiantes hayan experimentado por el PAIEP. Por esta razón, los resultados del estudio consideran fundamentalmente los cambios positivos.

\section{Generación de hábitos de estudio}

El cambio más aludido por los estudiantes en los talleres tuvo relación con los hábitos de estudio, particularmente con la percepción de haber adquirido y desarrollado técnicas $\mathrm{y}$ habilidades que les permitieron poco a poco gestionar de mejor manera las exigencias de la carrera. Como lo demuestra la literatura, una de las principales dificultades de la transición de la escuela a la universidad es que implica un salto significativo en cuanto al nivel de dificultad y exigencia académica, que en muchas ocasiones los estudiantes no se encuentran suficientemente preparados para enfrentar y que suele generarles un ánimo de desconcierto (CANALES; DE LOS RÍOS, 2007; SILVA; RODRIGUEZ, 2013).

En la mayoría de los casos, para resaltar la importancia de este cambio los estudiantes tendieron a aludir a sus experiencias escolares, en las cuales reconocen no haber necesitado mucha planificación ni uso de material de estudio adicional para que les fuera bien en las asignaturas. En el contexto de la educación media, entonces, en donde las exigencias académicas parecen haber sido con frecuencia bajas, el esfuerzo y la necesidad de generar mayor sistematicidad en la rutina de estudio también lo fueron, en la medida en que con eso bastaba para obtener buenas calificaciones.

Yo antes no tenía un hábito, estudiaba en el metro antes de llegar al colegio, estudiaba un poco, repasaba y me iba bien igual, y aquí no por, aquí hay que ir a clases, a los talleres, a las asesorías, y así llevar un ritmo. También tener un hábito de estudio en la casa, yo no era de los que llegaban a la casa a estudiar (Hombre, 18 años, ingeniería de ejecución en climatización, 30 de noviembre 2016.).

El ingreso a la universidad, de esta forma, fue vivido por los estudiantes como una experiencia que obligó a modificar sus estrategias de estudio y, particularmente, el modo en que organizaban su tiempo. Efectivamente, las nuevas exigencias académicas que trae consigo la universidad conllevan una mayor demanda de tiempo de estudio, porque -de acuerdo a como los estudiantes lo evidencian- la cantidad de los contenidos de las asignaturas no solo aumentan, sino que también se hacen más complejos que en el colegio.

Esta situación es descrita como un momento en el cual los estudiantes se ven sobrepasados y muchas veces paralizados ante las exigencias académicas universitarias, porque sienten que carecen de las herramientas de estudio para abordarlas. Por ello, cuando refieren al cambio relacionado con la generación hábitos que atribuyen a PAIEP, en algunos casos se señaló que más que mejorar la manera de estudiar, a través de los 
servicios de apoyo académico lograron generar nuevas maneras organizar el estudio y el tiempo que se le dedica a este.

Los hábitos de estudios más que mejorarlos, yo logré crear hábitos de estudio, porque yo no tenía nada, entonces llegar acá tratar de hacer calzar los horarios de clases, con las asesorías, entonces ahí uno se va ordenando y va creando más hábitos que los que ya tenía (Mujer, 19 años, bachillerato en ciencias y humanidades, 30 de noviembre 2016.).

Lo que hubo detrás del cambio en los hábitos de estudio fueron principalmente dos aspectos. En primer lugar, mencionan la adopción de ciertas estrategias de estudio que les permitieron hacer frente a la complejidad de los contenidos de sus asignaturas y organizar en acciones concretas el proceso de estudio. Se trata, ciertamente, de estrategias sencillas pero que permiten hacer más abordable los contenidos y estructuran el estudio como, por ejemplo, establecer etapas de desarrollo de ejercicios matemáticos, hacer resúmenes, generar esquemas, repasar la materia, entre otras cosas.

En segundo lugar, los estudiantes también señalan haber aprendido a planificar mejor su tiempo de estudio. En efecto, las exigencias académicas de la universidad demandan una mayor atención por parte de los estudiantes, modificando sus prioridades y el modo en que empleaban el tiempo hasta entonces. En esta medida, cuando los estudiantes ingresaron a la universidad se enfrentaron al desafío de organizar los diferentes dominios de su vida en función de las crecientes demandas de estudio del semestre, lo que significó una redistribución de su tiempo y de las actividades en que antes lo ocupaban. La universidad, en definitiva, fuerza a un cambio en el estilo de vida que tenían los estudiantes (REAY; CROZIER; CLAYTON, 2009). En ese contexto, los estudiantes señalan que en los servicios de apoyo académico recibieron las orientaciones necesarias para progresivamente ir compatibilizando el tiempo que les exige la universidad con los otros espacios de su vida.

Si se observa bien, se trata en ambos casos de maneras de abordar tanto el estudio como el tiempo que se le dedica a este, que no se encuentra formalizado ni tampoco tiene un carácter académico. Más bien, se trata de un conocimiento de naturaleza práctica, de un saber-hacer que proviene principalmente de la experiencia de los tutores, de lo que probablemente ellos también aprendieron cuando ingresaron a la universidad a partir de lo que les dio resultado y, por supuesto, de los consejos de otros. El cambio que experimentan los estudiantes, en ese sentido, es fruto de las orientaciones y recomendaciones que en el proceso de acompañamiento y durante el año les brindaron los tutores.

\section{Mejor comprensión de los contenidos de las asignaturas}

Luego de participar de PAIEP, los estudiantes también señalan haber experimentado una mejor comprensión de las asignaturas y de los contenidos tratados en clases. En los talleres evaluativos, fue una experiencia compartida por los estudiantes el haber enfrentado durante el inicio del primer año serias dificultades para comprender la materia que los profesores exponían, junto con sentir que en clases se avanzaba demasiado rápido y que comenzaban a quedarse rezagados en relación a sus compañeros. 
Esta barrera también ha sido identificada en otras investigaciones. En un estudio en Inglaterra sobre estudiantes de contexto de bajos ingresos que ingresaron a una universidad de élite, se evidencia que en ellos fue común la percepción de que su escolarización no proporcionó un acceso fácil a las formas de capital cultural dominantes en la educación superior (REAY; CROZIER; CLAYTON, 2009). De manera similar, en el caso de este estudio los estudiantes interpretaron esta barrera como la consecuencia de una preparación académica escolar insuficiente que, una vez en la universidad, les ha afectado en la comprensión de las asignaturas de la carrera y, colateralmente, en el desempeño académico, el cual dista mucho del que tuvieron en el colegio.

La percepción de haber sido insuficientemente preparados para la universidad, en algunos casos se hizo todavía más patente al constatar una brecha de conocimientos en relación con los compañeros que provenían de mejores colegios ${ }^{4}$. Aquello, ciertamente, constituye una evidencia en primera persona de los problemas de equidad del sistema educativo del país, en el cual los colegios presentan una alta diversidad en cuanto a la calidad de la enseñanza que entregan en función de los recursos con los que cuentan los establecimientos (AEQUALIS, 2011). Además, como lo han evidenciado otros estudios, este sentimiento de desigualdad frente a los compañeros constituye un factor que puede incidir en la decisión de abandonar la universidad (GONZÁLEZ, 2005).

[...] se me hizo súper difícil adaptarme porque tengo compañeros que vienen de liceos emblemáticos y todo y hay materia que en matemática me lo pasan y ellos lo hacen como 'ah sí, me lo pasaron en el colegio’ y yo nunca lo vi (Mujer, 19 años, ingeniería comercial, 25 de noviembre 2016.).

En este contexto, los servicios de apoyo académico de PAIEP cumplieron un importante rol en el proceso de aprendizaje que los estudiantes experimentaron en el primer año, especialmente en las asignaturas de mayor complejidad. Los estudiantes, así, expresaron el cambio que experimentaron por medio de la oposición entre dos momentos: uno inicial en el cual la comprensión de lo que el profesor exponía en clases era casi nula y en donde predominaba una sensación permanente de extravío, y otro posterior en el que gracias a la participación en los distintos servicios de apoyo académico, la materia comenzó gradualmente a hacer sentido, permitiéndoles ir involucrándose más en sus estudios.

El apoyo académico, en ese sentido, sirvió para entender mejor los contenidos y con ello comenzar a ajustarse al ritmo de avance que llevaban las asignaturas que, probablemente, se perdió al inicio del primer semestre. Sin embargo, en la medida en que los estudiantes al alero del acompañamiento académico van comprendiendo las asignaturas, también comienzan a darse cuenta de que también existieron formas de enseñar por parte de algunos docentes que no siempre contribuyeron al entendimiento de los contenidos. De esta manera, los estudiantes señalan que en ocasiones en clases

4- En Chile, la categoría de "liceo emblemático" se utiliza para referir a los colegios públicos tradicionales del país que seleccionan a sus estudiantes en función de su rendimiento académico. 
hubo contenidos que se pasaron rápidamente, ejercicios prácticos que fueron escasamente desarrollados y que no siempre existió disposición a aclarar dudas.

Nos ayudó harto en las evaluaciones más que nada, para hacer ejercicios porque la profe tampoco explica mucho, muy bien, si no sea por el PAIEP estaríamos muertos (Hombre, 19 años, química y farmacia, 28 de noviembre 2016.).

Aquello, ciertamente, responde a enfoques de enseñanza y aprendizaje anclados en una cultura académica tradicional que, como han apuntado otras investigaciones, eventualmente puede convertirse en un obstáculo para la integración de los estudiantes más desaventajados (SOBRERO et al., 2014). La contribución de PAIEP, en suma, no consiste únicamente en una nivelación en relación a los contenidos que los estudiantes no vieron en el colegio, sino también apoyar las dificultades que presentan los profesores para atender a un estudiantado con una preparación académica heterogénea.

Por último, otra forma en que se expresa el cambio de la mejor comprensión de los contenidos se relaciona con el desarrollo progresivo de una aproximación al aprendizaje de carácter profundo (BIGGS, 1989). En efecto, en ciertas ocasiones las instancias que brinda PAIEP permitieron a avanzar desde una orientación inicial de los estudiantes basada en la memorización de contenidos, a una búsqueda por comprender con algún grado mayor de profundidad los fundamentos de lo que se estudiaba.

A mí lo que principalmente me ayudó fue entender los ejercicios que hacía, porque yo al final los terminaba haciendo muy mecanizadamente, porque yo sabía qué hacer, pero no sabía el porqué, entonces si me cambiaban algo ya ahí me bloqueaba, eso fue lo principal que me ayudo (Hombre, 18 años, ingeniería de ejecución en climatización, 30 de noviembre 2016.).

Considerando todo lo expuesto, si recapitulamos e intentamos interpretar el cambio atribuido a los servicios académicos de PAIEP, desde el enfoque socio-cultural de VYGOTSKY (1978) y, particularmente, desde el concepto de zona de desarrollo próximo, los servicios proporcionan un puente entre el conocimiento y las habilidades con el cual los estudiantes ingresan a la universidad y las demandas académicas de las asignaturas de la carrera, permitiendo que gracias al acompañamiento de estudiantes más experimentados se comiencen a apreciar las relaciones entre lo que se les exige y lo que ya saben o pueden hacer (WOOD; WOOD, 1996). De esta forma, en el enfrentamiento ante materias que al comienzo les parecieron fuera del alcance a los estudiantes, la orientación de los tutores parece haber desempeñado un papel activo en el aprendizaje, contribuyendo a que progresivamente conectaran con los contenidos vistos en clases y fueran dominándolos.

\section{Recomposición de la confianza en sí mismo}

El tercer cambio positivo que los estudiantes señalan haber experimentado gracias a PAIEP se relaciona con el logro de mayor seguridad y confianza en sí mismos en relación a los estudios. Aquello se relacionó, ciertamente, con que en el primer semestre 
de universidad no lograron conseguir las mismas calificaciones, ni que les fuera tan bien como les iba previamente en el colegio. En el ingreso a la universidad, de esta forma, se removieron un conjunto de certezas basadas en el buen desempeño escolar asociadas a la autopercepción de siempre haber sido un buen estudiante. En otras palabras, la frustración de los malos resultados remeció una imagen de sí que los estudiantes construyeron en la etapa escolar, generando que enfrentaran con menor seguridad las evaluaciones y que cuestionaran sus capacidades para terminar la carrera.

En relación a ello, parte de la literatura sobre persistencia en educación superior ha sostenido que el desempeño académico previo influye significativamente en el desempeño futuro del estudiante ya que influye en el auto concepto, en la percepción de la dificultad de los estudios y en las expectativas de éxito (ETHINGTON, 1990). Asimismo, la evidencia en relación a la autoeficacia -entendida esta como la confianza de los estudiantes en su capacidad para llevar a cabo las tareas académicas- ha demostrado el efecto positivo que tiene en los indicadores de rendimiento universitario como la aprobación de créditos y el promedio de notas acumulado (ZAJACOVA; LYNCH; ESPENSHADE, 2005).

En la experiencia del primer año universitario, de esta forma, los estudiantes consideran que la participación en los servicios académicos de PAIEP les ayudó a recomponer la confianza y seguridad para enfrentar las asignaturas y sus evaluaciones. $\mathrm{Si}$ bien este cambio se encuentra estrechamente vinculado al analizado en el apartado anterior, en la medida en que la progresiva comprensión de las materias permite que los estudiantes vayan afianzándose; considera también una derivada más específica. En efecto, pues para muchos de los estudiantes fue común haber vivido una gran inseguridad antes y durante las evaluaciones, llegando incluso en los casos más extremos, a que el nerviosismo los bloqueara y no supieran qué responder en las pruebas. Aquello, sin duda, representó una experiencia de frustración que en el mejor de los casos significó un hecho aislado no tan difícil de superar, pero en los menos afortunados asentó un miedo que apareció con frecuencia al momento de las evaluaciones.

\footnotetext{
Después me fue yendo mejor, no sé, es que los unos o cuatro no existían en la [educación] media entonces me da como pena, como que me duele, 'yo pude haberlo hecho mejor', es que siempre hay algo que me pone nerviosa, sé hacerlo, pero como que lo veo y me bloqueo (Mujer, 18 años, química y farmacia, 25 de noviembre 2016.).
}

En ese sentido, lo que los estudiantes señalaron fue que la participación en los servicios y el apoyo de los tutores les permitió sentirse no solo más preparados en cuanto al estudio de los contenidos para rendir en las pruebas, sino también a afirmar la creencia de que pueden obtener mejores resultados. El cambio significó, de esta forma, el establecimiento de expectativas más positivas en relación al rendimiento en las evaluaciones, que permiten empezar a reconstruir aquella imagen del buen estudiante formada en el colegio y romper la racha de malas notas con la que se comenzó la universidad.

La confianza que me ha entregado PAIEP, como el tener mayores conocimientos que quedarme solamente con las cosas que nos daban y eso me daba confianza al momento de ir a las pruebas (Mujer, 19 años, bachillerato en ciencias y humanidades, 30 de noviembre 2016.). 
En suma, en este tercer cambio vivido por los estudiantes y atribuido a los servicios académicos, por tanto, se enfatiza una dimensión de este tipo de intervenciones que desde la literatura se suele identificar como una buena práctica, a saber, que se constituye en una instancia que permite a los estudiantes confiar en sus capacidades para enfrentar los desafíos de la universidad y mejorar su autoestima (TINTO, 2012).

\section{Obtención de un mejor rendimiento académico}

El cuarto cambio positivo que testimoniaron los estudiantes corresponde a la progresiva obtención de mejores resultados en las evaluaciones durante el primer año de universidad. En efecto, parte importante de los estudiantes que participaron en los talleres evaluativos señalaron que la asistencia a los servicios de apoyo académico de PAEIP trajo como consecuencia un mejor rendimiento académico, especialmente en las asignaturas que suelen reprobar más los estudiantes.

Para los estudiantes que asistieron con regularidad a PAIEP, de esta forma, no existen dudas en relación a si los servicios de apoyo académico ayudan a tener mejores notas. Aquello porque los buenos resultados no sólo se evidencian en sus propias calificaciones, sino también en las de los compañeros que asisten con frecuencia de las instancias del Programa. Sin dudas, eso constituye un incentivo para seguir asistiendo y aprovechar las instancias de reforzamiento académico.

Aunque puede parecer sencillo, si se considera en el contexto de los otros cambios que han sido analizados previamente, se puede constatar que el aumento del rendimiento académico tiene una significación profunda para los estudiantes. Ello pues, en primer lugar, viene a ratificar todos los cambios anteriores. Vale decir, sin mejores calificaciones resulta más difícil confirmar la efectividad de los nuevos hábitos de estudio, que se ha logrado una mejor comprensión de las asignaturas y, consecuentemente, apuntalar la confianza en uno mismo. Las mejores calificaciones obtenidas son, por consiguiente, la prueba que ratifica la existencia de los otros cambios y, probablemente, a partir de la cual estos se hacen perceptibles para los mismos estudiantes.

Creo que me ayudó a mejorar las notas, porque al principio yo me tropecé, me tropecé muy feo cuando llegué, yo no tenía nada de hábitos de estudio, porque a mí en el colegio me iba súper bien, pero no me esforzaba, entonces aquí llegué, y dije 'que tan diferente puede ser' y era todo muy diferente (Mujer, 19 años, bachillerato en ciencias y humanidades, 30 de noviembre 2016.).

En segundo lugar, el mejor rendimiento académico constituye la evidencia no solamente de la adquisición de los conocimientos y desarrollo de las competencias para resolver con buenos resultados una evaluación. Al mismo tiempo es una señal de que el mal desempeño del inicio comienza a dejarse atrás y comienza a constatarse que la universidad es un desafío difícil, pero ante el cual se tiene la capacidad de sobrellevar. Confirma, en definitiva, que los esfuerzos están dando frutos y se está a la altura para rendir en la universidad. 
Los cambios que más destaco son respecto a mi rendimiento y creo que sin la ayuda de PAIEP no hubiera sido igual, como yo vengo de un colegio técnico [...] nos enseñaban un cuarto de la materia que nos deberían enseñar, entonces eso encuentro que gracias a PAIEP he podido salir adelante en la universidad (Mujer, 19 años, bachillerato en ciencias y humanidades, 1 de diciembre 2016.).

La consecución de mejores calificaciones, en este contexto, tiene para los estudiantes un significado más hondo que el de una mera estrategia orientada a aprobar las asignaturas a como dé lugar. Aunque aquello no deja de tener legitimidad, como se ha podido evidenciar, para los estudiantes la mejora en el rendimiento académico que han alcanzado y en el que PAIEP contribuyó, es la confirmación de dos elementos de gran importancia. Por un lado, representan una señal de que los cambios que ha requerido el ajuste al contexto universitario van en la dirección correcta, pues han conseguido buenos resultados. Por otro lado, representan un envión anímico por medio del cual los estudiantes, especialmente si es que en algún momento lo pusieron en duda, consiguen certezas de que sirven para lo que están estudiando.

\section{Contribución a la integración social}

Finalmente, el último cambio expresado por los estudiantes estuvo relacionado con el desarrollo de ciertas habilidades blandas que contribuyeron a la integración. La importancia de este cambio debe leerse en el contexto de lo que distintos estudios sobre inclusión en educación superior han demostrado, en relación a las dificultades de integración social que los estudiantes que provienen de sectores vulnerables experimentan al ingresar a la universidad. En ese sentido, si para todos quienes ingresan a la universidad significa lidiar con una institución desconocida que implica romper con sentidos del mundo familiar y escolar previo, el establecimiento de vínculos sociales pareciera ser algo más difícil para el caso de los estudiantes que asisten a PAIEP.

En efecto, para el caso de los estudiantes que accedieron mediante el Propedéutico ciertas investigaciones evidenciaron que al término del primer año fueron menos participativos en clase, tuvieron menos interacción con sus compañeros (tanto dentro como fuera de clase) y, además, que hicieron menos preguntas en clase y presentaciones que quienes ingresaron por admisión regular (KOLJATIC; SILVA, 2010). Otros estudios señalan que la desventaja que sentían al comienzo con relación a sus compañeros fue algo que dificultó el establecimiento de vínculos de sociabilidad (TREVIÑO; SCHEELE; FLORES, 2014). Aquello también ha sido evidenciado en otros países, pues estudios sobre universidades mexicanas han mostrado la dificultad para relacionarse con desconocidos y acostumbrarse a las nuevas formas de convivencia, así como también dificultades para hacer amigos (SILVA; RODRÍGUEZ, 2013).

En este contexto, el rol que tuvo PAIEP en el ingreso de los estudiantes a la universidad fue clave, ya que en sus relatos emerge con un soporte fundamental. Así, los estudiantes describen el Programa como un lugar en el cual siempre hubo disposición para ayudarlos, preocupación por ellos y orientación en lo que necesitaran, tanto por 
parte de los tutores como de los profesionales. Aquello, sin dudas, genera una sensación de acogida, de buen recibimiento que fomenta el sentido de pertenencia de los estudiantes a la institución.

Me ha ayudado personalmente a mí a ser una persona más abierta, por lo menos una vez al mes ellos nos hacen un seguimiento, una entrevista de cómo nos ha ido, entonces yo por eso he podido romper un poco esa barrera del retener todo para mí, sino que soltar un poco, contar mis cosas e ir contando cómo me ha ido en la universidad (Mujer, 20 años, bachillerato en ciencias y humanidades, 1 de diciembre 2016.).

Así, es posible apreciar que si bien el acompañamiento que brinda PAIEP a los estudiantes -de acuerdo a cómo fue diseñado por los encargados- tiene una orientación principalmente académica debido a que el objetivo último es que los estudiantes no abandonen la universidad por razones vinculadas al rendimiento académico, existe una dimensión psicosocial de los apoyos que es de gran importancia. En efecto, la preocupación constante por saber cómo les ha ido a los estudiantes, aunque se centra en lo académico no deja de lado los aspectos más personales que viven en la transición, lo que los hace sentir acompañados en esta etapa entregándoles confianza y seguridad.

El papel que cumple PAIEP en este cambio es contribuir, a través de sus servicios, a que los estudiantes poco a poco vayan aprendiendo a sobrellevar los desafíos del primer año (la frustración ante las malas notas, el estrés que generan las exigencias académicas, la ansiedad, frente a las evaluaciones, entre otras) y comiencen a sentirse más cómodos en la universidad.

Me han cambiado mi forma de ver, el cómo enfrentar el proceso a lo largo del año, venía con un poco de temor, con un poco de miedo al fracaso y pucha el apoyo psicosocial para mí ha sido muy importante, porque tanto el apoyo grupal e individual me ha servido mucho (Hombre, 21 años, tecnología en administración de personal, 1 de diciembre 2016.).

En síntesis, el PAIEP es concebido como una instancia de apoyo académico y social fundamental en la permanencia de los estudiantes de menores ingresos y/o que vienen menos preparados académicamente para la universidad. Efectivamente, la importancia de los organismos como PAIEP que generan medidas de acompañamiento radica en que fungen como una puerta de entrada en la cual se les entrega el conocimiento que necesitan para navegar con mayor seguridad en el terreno desconocido de la universidad (TINTO, 2008).

\section{Discusión}

El estudio presentado representa un esfuerzo por explorar, a través de una metodología de evaluación poco conocida e innovadora, los cambios más importantes que percibieron los estudiantes de primer año de la Universidad de Santiago que participaron de las distintas instancias de apoyo académico que brinda el PAIEP. El recorrido por el análisis de los 
cambios atribuidos al PAIEP que se ha realizado permite generar reflexiones tanto a nivel de la intervención y el logro de la permanencia, como de la metodología empleada.

En cuanto a lo primero, los cambios experimentados durante el primer año universitario y que los estudiantes atribuyeron a la participación en el PAIEP pueden ser interpretados como una contribución al proceso de afiliación a la educación superior y de adquisición del oficio de estudiante (COULON, 2017). De acuerdo con el autor, el graduado de la educación secundaria debe aprender a convertirse durante el primer año de universidad en estudiante, un verdadero proceso de transformación por el cual se adquiere un nuevo estatus social. El nuevo estudiante, así, debe incorporar rutinas, evidencias, reglas y nuevos códigos universitarios; ir afiliándose progresivamente al mundo intelectual en el que se ha ingresado, la más de las ocasiones sin un conocimiento real. La permanencia y el éxito en la universidad de quienes recién ingresan, en ese sentido, se juega en detectar, descifrar y luego incorporar estos códigos por medio de los cuales se adquiere el oficio de estudiante.

En este marco, los servicios que brinda PAIEP constituyen un puente que aproxima a los estudiantes -que probablemente más lo requieren- a aquel oficio. Ayudan a acortar la distancia experimentada en un inicio con el medio universitario, a adquirir paulatinamente las reglas y códigos que lo rigen. En efecto, la generación de los hábitos de estudio necesarios para abarcar y organizar la complejidad y profundidad de las asignaturas de la carrera; la mejor comprensión de los contenidos de las clases; la recomposición de la confianza en sí mismo luego del fracaso experimentado en las primeras pruebas; la obtención de mejores calificaciones que confirma la adquisición de los códigos universitarios y, finalmente, la contribución a la integración social, representan cinco cambios en ámbitos específicos en los que permitieron a los participantes comenzar a adquirir el oficio de estudiante y ejercerlo con mayor naturalidad.

En relación a la metodología utilizada en el estudio, es posible sostener que esta permitió identificar un conjunto de cambios atribuidos a la intervención, que si bien pueden parecer esperados, tienen el valor de sistematizar, articular y priorizar aquellos que los estudiantes consideraron más relevantes. Además, la metodología permitió capturar cambios emergentes que experimentaron los estudiantes, posibilitando relevar no solo aquellos relacionados con el ámbito académico (hábitos de estudio, comprensión de los contenidos y el rendimiento), sino también otros relacionados con aspectos subjetivos (como la confianza en sí mismo) y sociales (como la integración).

Sin embargo, la limitación que es posible encontrar en cuanto a la metodología, se relaciona con los cambios negativos de la intervención estudiada, frente a los cuales los estudiantes no dieron cuenta de ninguno. Sin rechazar la hipótesis de que efectivamente los estudiantes no hayan experimentado ningún cambio negativo, es plausible considerar ciertas características del dispositivo metodológico implementado que podrían haber inhibido que se manifestaran. En primer lugar, cabe considerar que la estrategia de selección de los informantes por caso extremo haya conllevado una suerte de sesgo de selección y que como los estudiantes que participaron del estudio asistieron con una alta frecuencia a los servicios de apoyo académico, hayan estado más predispuestos a hablar sobre todo de los aspectos positivos del programa. En segundo lugar, también se considera como un posible factor inhibidor el hecho de que los cambios hayan sido recogidos en instancias grupales, 
pues la grupalidad tiende a suspender el posible y radical disenso (CANALES, 2006). Por último, la técnica del cambio más significativo recoge experiencias de las cuales los actores son conscientes y pueden expresar, en tal caso cabe preguntarse si los cambios negativos pudiesen formar parte de un registro más difícil de articular y verbalizar.

De todas formas, parece ser importante que los estudiantes no hayan mencionado como cambios haber experimentado ningún tipo de discriminación o dificultades para establecer relaciones a causa de su participación en PAIEP, como algunos estudios lo han reportado en relación al Propedéutico (TREVIÑO; SCHEELE; FLORES, 2014). Si bien aquello puede considerarse una buena noticia en la medida en que significa un avance en la instalación de políticas y prácticas inclusivas; también es cierto que la moderación de los talleres evaluativos realizados estuvo orientada a capturar especialmente los cambios que emergieran de los propios estudiantes.

En ese sentido, los desafíos para las investigaciones que busquen profundizar tanto en los resultados de la investigación como el uso del CMS en la evaluación de programas para la permanencia, son principalmente dos. Por un lado, incluir otras categorías de participantes que puedan iluminar tanto los cambios positivos como negativos de las intervenciones, como estudiantes que hayan tenido una baja asistencia al programa, renunciado a este o, incluso, abandonado la universidad. Por otro, profundizar especialmente en las consecuencias no esperadas de los programas, no tanto con el objetivo de encontrar lo que la intervención hace mal, como de garantizar que efectivamente no genera cambios negativos.

\section{Referencias}

AEQUALIS. Propuestas para la educación superior: foro aequalis y las transformaciones necesarias. Santiago de Chile: Inacap, 2011.

BEAN, John. Dropouts and turnover: the synthesis and test of a causal model of student attrition. Research in Higher Education, Holanda, v. 12, n. 2, p. 155-187, 1980.

BENSIMON, Estela. The underestimated significance of practitioner knowledge in the scholarship of student success. Review of Higher Education, EEUU, v. 30, n. 4, p. 441-469, 2007.

BIGGS, John. Approaches to the enhancement of tertiary teaching. Higher Education Research and Development, Australia, v. 8, n. 1, p. 7-25, 1989.

CANALES, Andrea; DE LOS RÍOS, Danae. Factores explicativos de la deserción universitaria. Revista Calidad en la Educación, Santiago de Chile, n. 26, p. 173-201, jul. 2007.

CANALES, Manuel. Metodologías de investigación social. Santiago de Chile: Lom, 2006.

CARREÑO, Beatriz; MICIN, Sonia; URZUA, Sergio. Una caracterización inicial para el logro académico de estudiantes de primer año universitario. Cuadernos de Investigación Educativa, Santiago de Chile, v. 7, n. 1, p. 29-39, jun. 2016. 
COULON, Alain. Le métier d'étudiant: I'entrée dans la vie universitaire. Paris: Presses Universitaires de France, 1997.

COULON, Alain. Le métier d'étudiant: I'entrée dans la vie universitaire. Educação e Pesquisa, São Paulo, v. 43, n. 4, p. 1239-1250, dic. 2017.

DUBET, François. Dimensions et figures de l'expérience étudiante dans l'université de masse. Revue française de sociologie, París, v. 35, n. 4, p. 511-532, 1994.

CRISP, Gloria; CRUZ, Irene. Mentoring college students: A critical review of the literature between 1990 and 2007. Research in Higher Education, EEUU, v. 50, n. 6, p. 525-545, abr. 2009.

DAVIES, Rick; DART, Jessica. The 'Most Significant Change' (MSC) Technique: a guide to its use. London: CARE International, 2005. Disponible en: <http://www.mande.co.uk/docs/MSCGuide.pdf>. Acceso en: 10 sept. 2015.

ETHINGTON, Corinna. A psychological model of student persistence. Research in Higher Education, EEUU, v. 31, n. 3, p. 266-269, jun. 1990.

FELOUZIS, Georges. La condition étudiante: sociologie des étudiants et de l'université. París: Presses Universitaires de France, 2001.

GERSHENFELD, Susan. A Review of undergraduate Mentoring Programs. Review of Educational Research, EEUU, v. 84, n. 3, p. 365-391, sept. 2014.

GONZÁLEZ, Luis. Estudio sobre la repitencia y deserción en la educación superior chilena. Santiago de Chile: [s. n.], 2005. Disponible en: <https://www.inacap.cl/tportal/portales/tp4964b0e1bk102/uploadlmg/ File/REPITENCIA_DESERCION_L_E_Gonzalez_2005.pdf>. Acceso en: 24 nov. 2017.

JACOBI, Maryann. Mentoring and undergraduate academic success: a literature review. Review of Educational Research, EEUU, v. 61, n. 4, p. 505-532, dic. 1991.

KOLJATIC, Mladen; SILVA, Mónica. Algunas reflexiones a siete años de la implementación de la PSU. Revista Estudios Públicos, Santiago de Chile, n. 120, p. 125-146, 2010.

OCDE - Organización para la Cooperación y el Desarrollo Económicos. La educación superior en Chile. Santiago de Chile: OCDE, 2009. Disponible en: <http://www.oecdilibrary.org/laeducacion-superior-enchile_5ksntst3t7hd.pdf>. Acceso en: 03 oct. 2016.

PASCARELLA, Ernest; TERENZINI, Patrick. Predicting freshman persistence and voluntary dropout decisions from a theoretical model. The Journal of Higher Education, EEUU, v. 51, n. 1, p. 60-75, 1980.

RAHMER, Beatriz; MIRANDA, Rafael; GIL, Francisco. Programa de acceso inclusivo equidad y permanencia de la Universidad de Santiago de Chile: una política universitaria de acción afirmativa. In: CONFERENCIA LATINOAMERICANA SOBRE ABANDONO EN LA EDUCACIÓN SUPERIOR (CLABES), 3., 2013, México, DF. Publicación de la... México, DF: Clabes, 2013. p. 1-13. 
REAY, Diane; CROZIER, Gill; CLAYTON, John. Strangers in paradise? Working-class students in elite universities. Sociology, London, v. 43, n. 6, p. 1103-1121, dic. 2009.

SCHEELE, Judith. Logros y desafíos pendientes para la inclusión y retención en la educación superior. Santiago de Chile: Centro de Políticas Comparadas de Educación: UDP, 2015. Disponible en: <http://cpce. udp.cl/wp-content/uploads/2016/08/IPE7.pdf>. Acceso en: 7 sept. 2016.

SCRIBANO, Adrián. El proceso de investigación social cualitativo. Buenos Aires: Prometeo, 2008.

SILVA, Marisol. El primer año universitario: un tramo crítico para el éxito académico. Perfiles Educativos, México, DF, v. 33, p. 102-114, 2011.

SILVA, Marisol; RODRÍGUEZ, Adriana. ¿Cómo viven el primer año los jóvenes provenientes de sectores de pobreza? In: GUZMÁN, Carlota. Los estudiantes y la universidad: integración, experiencias e identidades. México, DF: Anuies, 2013. p. 95-128.

SOBRERO, Viviana et al. Equidad y diversidad en universidades selectivas: la experiencia de estudiantes con ingresos especiales en las carreras de salud. Pensamiento Educativo, Santiago de Chile, v. 51, n. 2, p. 152-164, 2014.

STRAUSS, Anselm; CORBIN, Juliet. Bases de la investigación cualitativa: técnicas y procedimientos para desarrollar la teoría fundamentada. Antioquía: Universidad de Antioquía, 2002.

TINTO, Vincent. Access without support is not opportunity. In: THE ANNUAL INSTITUTE FOR CHIEF ACADEMIC OFFICERS, 36., 2008, Washington, DC. Anais... Washington, DC: [s. n.], 2008. p. 1-9.

TINTO, Vincent. Completing college: rethinking institutional action. Chicago: Chicago University Press, 2012.

TINTO, Vincent. Leaving college: rethinking the causes and cures of student attrition. Chicago: Chicago University Press, 1993.

TREVIÑO, Ernesto et al. Estudio sobre transiciones educativas en la enseñanza media y definiciones de la política para este nivel educativo. Santiago de Chile: Centro de Políticas Comparadas de Educación, 2016.

TREVIÑO, Ernesto; SCHEELE, Judith; FLORES, Stella. Beyond the test score: a mixed methods analysis of a college access intervention in Chile. Journal of Mixed Methods Research, EEUU, v. 1, n. 11, p. 255-265, abr. 2014.

VYGOTSKY, Lev. El desarrollo de las funciones psicológicas superiores. Barcelona: Grijalbo, 1978.

WARREN, Digby. Curriculum design in a context of widening participation in higher education. Arts and Humanities in Higher Education, London, v. 1, n. 1, p. 85-99, 2002.

W00D, David; W00D, Heather. Vygotsky, tutoring and learning. 0xford Review of Education, Oxford, v. 22, n. 1, p. 5-16, jun. 1996. 
ZAJACOVA, Anna; LYNCH, Scott M.; ESPENSHADE, Thomas. Self-efficacy, stress, and academic success in college. Research in Higher Education, EEUU, v. 46, n. 6, p. 677-706, sept. 2005.

Recibido en: 26.01.2018 Revisiones en: 24.04.2018 Aprobado en:13.06.2018

Rodolfo Martinic Lenta es sociólogo en la Pontificia Universidad Católica de Chile, Máster en Psicología Social en la Universidad Diego Portales de Chile y actualmente doctorante en Sociología en la Ecole des Hautes Etudes en Sciences Sociales, Francia. Durante tres años se desempeñó como investigador en el área de Evaluación de Procesos Educativos de la Universidad de Santiago de Chile. 